DOI: $10.3901 / J M E .2020 .12 .133$

\title{
高速列车底部流动特性分析*
}

\author{
朱剑月 $1,2,3$ 吕苏 ${ }^{1,3}$ 陈力 ${ }^{1}$ 沈 哲 ${ }^{1}$ \\ (1. 同济大学上海地面交通工具风洞中心 上海 201804; \\ 2. 大功率交流传动电力机车系统集成国家重点实验室 株洲 412001; \\ 3. 同济大学铁道与城市轨道交通研究院 上海 201804)
}

\begin{abstract}
摘要: 高速列车底部流动是形成道砟飞砟的重要原因。基于延迟分离浴模型计算模拟了包含地面的高速列车底部流动 特性, 结果表明, 由于流动分离与湍治发展, 列车周围 (尤其在车头、转向架、车厢连接处与尾车等部位) 产生了高湍 流度非定常流动。车头部位产生的强压力脉动, 极易诱发道砶颗粒在列车风作用下产生飞砟。列车底部流场内, 与钢 轨踏面以下区域相比, 踏面以上部位流动湍流发展充分, 强度较高, 使得道砟颗粒在轮轨动力作用下振离道床面后, 易于在流场脉动力作用下产生飞砟。铰接式转向架区域流场, 受到车厢连接部位流动干扰, 产生较多涡流, 湍流强度 较大。列车尾迹内形成的大尺度尾浴下洗扫掠道床面形成较强压力脉动, 容易导致道磴颗粒被列车风卷起, 散落至钢 轨面上。因此, 车头流线型设计、平顺车厢连接部位与控制列车尾流发展, 将有利于降低列车底部流动对于道床面作 用、减少飞砟现象发生。
\end{abstract}

关键词: 高速列车; 列车空气动力学; 道砟飞砟；延迟分离浴模拟；流动特性 中图分类号: U270

\section{Investigation on the Characteristics of the Underbody Flow Around High-speed Train}

\author{
ZHU Jianyue ${ }^{1,2,3} \quad \mathrm{LÜ} \mathrm{Su}^{1,3}$ CHEN Li ${ }^{1} \quad$ SHEN Zhe
}

(1. Shanghai Automotive Wind Tunnel Center, Tongji University, Shanghai 201804;

2. The State Key Laboratory of Heavy Duty AC Drive Electric Locomotive Systems Integration, Zhuzhou 412001;

3. Institute of Rail Transit, Tongji University, Shanghai 201804)

\begin{abstract}
The turbulent underbody flow developed around a high-speed train is the crucial factor for ballast flight. Based on the delayed detached-eddy simulation, the flow behaviour around a scaled high-speed train with ground underneath is investigated. Results show that due to flow separations and vortex interactions, the highly unsteady flow is generated around the train, especially at the regions of the nose, the bogies, the inter-carriage gaps and the tail car. A significant pressure pulse produced at the nose area is expected to play a key role in the initiation of the ballast flight and make it happen more easily. Compared to the areas underneath the rail running surface, the flow fluctuations above the rail are larger. Thus, the ballast particles are conductive to be projected by the fluctuating flow beneath the train after separated from the trackbed surface under the strong wheel-rail excitement. Due to the special location within the inter-car gaps, the strong vortical flow is developed and distributed around the articulated bogie region. The train tail generates the large trailing vortices within the train wake which act on the ballasted trackbed surface and may induce the ballast flight. Therefore, the streamlined design of the nose, the smoothing of the inter-carriage gaps and the control of the train wake, which can reduce the interaction between the train underbody flow and the trackbed surface, and thus reduce the occurring of the ballast flight.
\end{abstract}

Key words: high-speed train; train aerodynamics; ballast flight; delayed detached-eddy simulation; flow behavior

* 国家自然科学基金 (51875411)、上海市专业技术服务平台（19DZ2290400） 和甡引动力国家重点实验室开放课题 (TPL1804) 资助项目。20191018 收 到初稿, 20191231 收到修改稿 


\section{0 前言}

高速铁路在世界范围内发展迅猛, 道砟飞砟现 象在高速与快速有䃇轨道线路上经常发生, 对列车 与线路产生较大破坏作用。飞溅的道砟颗粒会击打 列车轮轴而导致损伤, 若散落在钢轨踏面上, 在剧 烈的轮轨载荷作用下, 造成钢轨核伤与损伤, 从而 加剧轮轨动力作用, 加快轨道状态恶化。国外较多 国家高速铁路轨道结构采用碎石道床, 虽然我国高 速铁路多数采用无砤轨道结构, 但大量的既有线路 铺设了有碴轨道结构, 随着列车运行速度的不断提 高, 道砟飞砟问题不容忽视。

关于高速列车空气动力学性能, 众多学者进行 了分析研究。田红旗 ${ }^{[1]}$ 总结了我国高速铁路发展进 程中, 高速轨道交通空气动力学研究形成的理论、 技术与所解决的科学问题。杨国伟等 ${ }^{[2]}$ 系统阐述了 我国高速列车研制和运行过程中关键力学问题(包 括高速列车空气动力学) 的研究。梁习锋等 ${ }^{[3]}$ 通过风 洞试验研究了列车外形对列车气动性能的影响。

BAKER 等 ${ }^{[4-5]}$ 基于数值模拟与现场测试, 分析了高 速列车周围流场的气动特性。QUINN 等 ${ }^{[6]}$ 认为高速 列车长细比较大、近地运行且底部结构复杂, 道砟 飞砟现象的产生与列车底部流动密切相关, 且是由 于车体周围流动与轨道结构振动共同作用的结果, 其形成过程具有随机性。GARCIA 等 ${ }^{[7]}$ 根据 COUETTE 流动建立了简化模型, 分析了高速列车 底部与地面间流动基本特性, 认为道床表面剪应力 是诱发道碴颗粒飞溅的重要因素。孙振旭等 ${ }^{[8]}$ 采用 定常方法计算分析了地面效应对高速列车气动力的 影响, 认为地面边界层、列车与来流方向间侧偏角、 列车与轨面相对高度等参数将影响高速列车气动力 性能。ZHANG 等 ${ }^{[9]}$ 建立了高速列车 3 车编组比例模 型, 计算分析了近地转向架区域流场与气动力特性, 并进行了风洞试验验证。BELL 等 ${ }^{[10]}$ 在风洞内建立 了碎石道床模型, 通过钢轨支承列车模型, 分析了 列车周围流动发展与尾浴结构特性, 以揭示列车尾 流内最大列车风产生机理。PREMOLI 等 ${ }^{[11]}$ 通过风 洞测试认为增加道床面与轨枕顶面间距可以减少道 砟飞砟发生, 而扁平状道碴颗粒容易被列车风吹起。 ZHU 等 ${ }^{[12]}$ 模拟计算了气流经由转向架舱下有砟轨 道面时道砟颗粒的气动特性, 认为碎石道床作为多 孔介质松散体结构, 颗粒之间的间隙和连通效应会 增加道砟颗粒气动力作用, 促使飞砟现象发生。 $\mathrm{ZHU}$ 等 ${ }^{[13]}$ 基于数值计算发现转向架后轮对与转向
架舱后壁区域通过道床面时, 道砟飞砟容易产生; 当道砟颗粒离开道床面后, 将受到较大气动力作用。 WANG 等 ${ }^{[14]}$ 通过组合车轮旋转与地面移动状态形 成不同工况，基于数值模拟计算分析了列车周围流 动与地面边界层的相互作用, 发现地面边界层生成 与发展将直接影响列车风速度较高区域幅值与位 置, 并使得列车尾浴形状改变而加宽尾迹, 且车轮 旋转对列车底部流动影响主要集中在转向架区域, 对于列车风和尾流影响较小。

综上所述, 正确理解高速列车底部流动特征是 分析道砟飞砟成因的关键, 由于高速列车结构复杂, 尤其近地转向架区域包含诸多结构部件, 以往研究 多数采用简化或 3 车编组模型, 采用定常或壁面函 数法求解高速列车周围流场并进行列车底部流动分 析。本文将在前期研究基础上，通过建立同时采用 普通和铰接式转向架结构的高速列车 6 车编组模 型, 基于延迟分离涡模型(Delayed detached-eddy simulation, DDES)进行高速列车固壁表面边界层求 解与列车周围流场数值计算, 以准确模拟高速列车 底部与道床面之间高湍流度的复杂非定常流动, 为 揭示道砟飞砟现象产生机理建立理论基础。

\section{1 数值计算方法}

高速列车周围为低马赫数流动, 在计算流体动力 学性能时, 可忽略流体的可压缩性。采用开源软件 OpenFOAM 求解不可压缩流体动力学控制方程, 空间 和时间离散采用二阶精度格式, 基于 Spalart-Allmaras (S-A) 湍流模型的延迟分离浴模型用来进行数值模 拟 ${ }^{[15]}$ 。S-A 模型作为单方程模型, 采用涡黏系数 $\tilde{v}$ 求 解对流-扩散方程 ${ }^{[16]}$, 其输运方程定义为

$$
\begin{gathered}
\frac{\partial \tilde{v}}{\partial t}+\boldsymbol{u}_{j} \frac{\partial \tilde{v}}{\partial x_{j}}=G_{\tilde{v}}-Y_{\tilde{v}}+ \\
\frac{1}{\sigma_{\tilde{v}}}\left[\frac{\partial}{\partial x_{j}}\left((v+\tilde{v}) \frac{\partial \tilde{v}}{\partial x_{j}}\right)+C_{b 2}\left(\frac{\partial \tilde{v}}{\partial x_{i}}\right)\left(\frac{\partial \tilde{v}}{\partial x_{i}}\right)\right]
\end{gathered}
$$

式中, 方程右边的三项分别为浴黏系数 $\tilde{v}$ 的生成项 $\left(G_{\tilde{v}}\right)$, 耗散项 $\left(Y_{\tilde{v}}\right)$ 和扩散项; $v$ 为运动黏性系数; $C_{b 2}$ 为湍流模型经验常数; $x_{i}$ 表示笛卡儿坐标系的三个方 向 $(i=1,2,3) ; u_{j}$ 为三方向流体速度分量 $(j=1,2,3)$ 。

作为混合方法, 分离涡模型 (Detached-eddy simulation, DES)在几何结构附近边界层区域内采用 基于雷诺平均(Reynolds average Navier-Stokes, RANS)的模型获取小尺度湍流信息，而在流动分离 区域内应用大浴模拟(Large eddy simulation, LES) 求解大尺度湍流结构, 通过比较网格尺度与湍流尺 度大小来实现 RANS 至 LES 的转换。为避免 “网格 
诱导分离”, 延迟分离涡模型通过引入过渡函数推迟 RANS 至 LES 的转换, 其湍流长度尺度 $\tilde{d}$ 定义为

$$
\tilde{d} \equiv d-f_{d} \max \left(0, d-C_{\mathrm{des}} \Delta\right)
$$

式中，网格间距 $\Delta=\max (\Delta x, \Delta y, \Delta z)$, 取决于计算网 格在 $x 、 y$ 和 $z$ 三方向上的最大间距; 经验常数 $C_{d e s}=0.65$, 由均匀湍流计算获得; $d$ 为流场点距 壁面距离; 延迟函数 $f_{d}$ 定义为

$$
f_{d} \equiv 1-\tanh \left(\left(8 r_{d}\right)^{3}\right)
$$

式中, 参数 $r_{d}$ 为应用于浴黍模型中的长度尺度比, 可表示为

$$
r_{d} \equiv \frac{v_{t}+v}{\sqrt{U_{i, j} U_{i, j}} \kappa^{2} d^{2}}
$$

式中, $v_{t}$ 为湍流动浴黏度; $U_{i, j}=\partial U_{i} / \partial x_{j}$ 是速度梯 度; $\kappa$ 为卡门常数。延迟函数 $f_{d}$ 在 LES 求解域内设 计为 1 而在其余区域为 0 , 使得湍流长度尺度 $\tilde{d}$ 通 过设置 $f_{d}$ 为 0 与 $d$ 相等激发 RANS 模块。

\section{2 列车计算模型}

欧洲多数国家高速铁路普遍采用有砟轨道结 构, 故根据 “欧洲之星” 高速列车原型建立 $1: 25$ 比例模型, 转向架区域内各部件进行适当简化, 由 于研究车体底部流动, 为节省计算资源忽略受电弓 结构。该列车模型为 6 节编组, 具有不同转向架结
构和车厢间间距, 沿列车纵向前后对称布置两单元, 每单元包含一头车(或尾车)和采用铰接式转向架联 接的两中间车, 其余转向架均为普通转向架, 如 图 1 所示 ${ }^{[17]}$ 。两列车单元间车厢连接部位的间距 较其余车厢连接部位大。由于列车计算模型编组 较多, 沿纵向长度较长, 故数值分析采用的计算 域(图 2)沿流向、垂向和展向的尺寸分别设为 $100 H, 16 H$ 与 $26 H(H$ 为列车高度), 使得计算域 阻塞比为 $0.1 \%$, 远低于 $\mathrm{CEN}$ (欧洲标准委员会)的 建议值 $1 \%^{[18]}$, 以确保计算域顶面、两侧面与出口 等边界与列车模型保持足够远距离, 从而对列车 周围流动发展产生的影响降到最小程度。考虑数 值风洞模型, 列车静止在来流中, 计算域边界条 件设置为: 来流为低湍流度均匀流 $\left(U_{\infty}=40 \mathrm{~m} / \mathrm{s}\right)$; 顶面与两侧面均设为剪切为零的滑移壁面; 出口 定义为压力出口; 车体各几何体表面和地面均设 置为静态无滑移壁面。

基于文献[19]的网格生成技术和网格无关性分 析, 采用相同网格划分软件 Hexpress 和网格拓扑方 法在计算域内生成约 1.2 亿个非结构网格, 车头区 域几何体周围网格如图 3a 所示, 几何体表面采用三 角形和四边形网格划分, 通过按增长率 1.1 向外拉 伸生成棱柱形边界层网格, 经由四面体网格过渡到 体域网格。计算模型考虑轮轨接触, 为便于网格划

图 1 高速列车模型 (6 车编组)
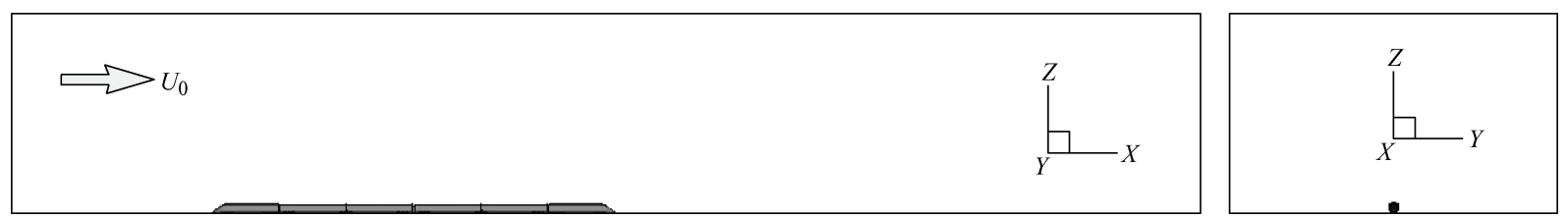

图 2 列车整体计算域 (按比例缩放)

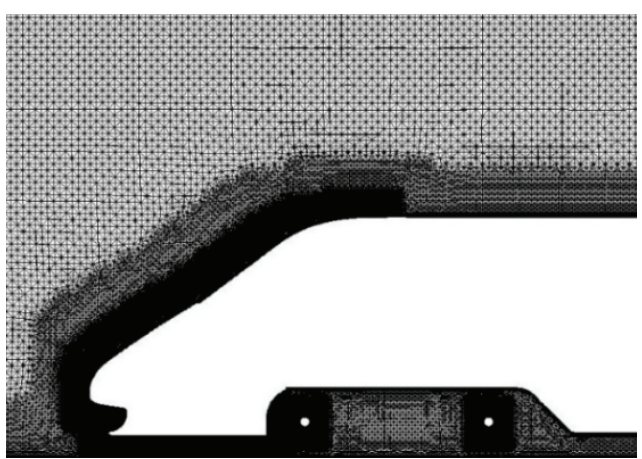

(a) 车头沿流向中心面网格划分

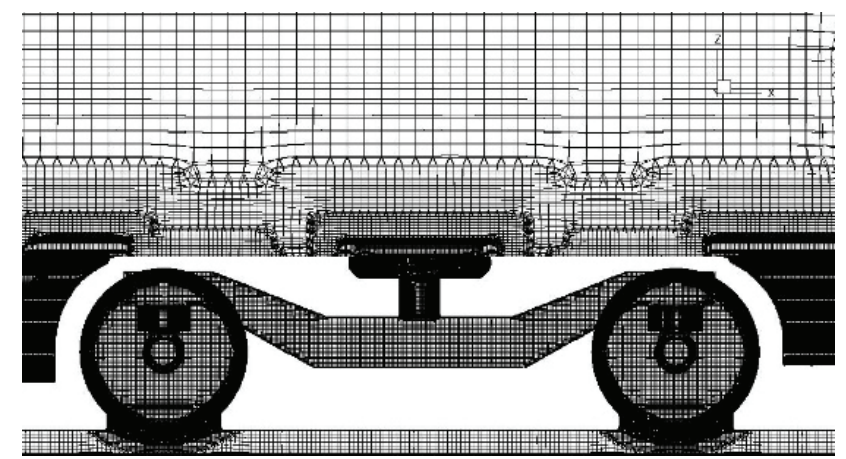

(b) 轮轨接触区域网格划分

图 3 计算模型网格划分 
分与提高网格质量, 适当加大了轮轨间接触面积, 该区域网格划分如图 3b 所示。边界层划分为 15 层, 第一层网格至列车壁面距离为 $1.5 \times 10^{-5} \mathrm{~m}$ 并沿着壁 面法矢量方向以 1.2 的增长率向外拓展, 使得 $y^{+}$(第 一层网格质心到壁面的无量纲距离, $y^{+}=y u_{\tau} / v$, 其中 $y$ 是网格质心至壁面距离, $u_{\tau}$ 为摩擦速度, $v$ 为 运动黏性系数) 的最大值不超过 3 , 以确保边界层准确 求解以及所采用的湍流模型在黏性底层内考虑低雷诺 数效应。数值仿真时间步长采用 $2.5 \times 10^{-6} \mathrm{~s}$, 使得 Courant-Friedrichs-Lewy (CFL) 数在大部分计算区域内 小于 1 而在整个计算区域内的最大值不超过 $8, \mathrm{CFL}$ 较高值仅出现在车体结构产生几何突变的尖缘部位。

\section{3 计算结果分析}

数值模拟在大型并行机 Iridis4 (University of Southampton) 上进行, 计算时采用 480 个处理器, 运行 $2.3 \times 10^{5}$ 个时间步, 流场达到统计意义上稳定, 再 运行 $2.7 \times 10^{5}$ 个时间步进行数据采集, 以使计算结果的 频谱分析获得合理的频率分辨率。以下数值计算结果 中, 对列车底部物面附近流场内压力与速度系数、 $Q$ 准则等值面、浴量场、湍流强度与转向架气动力等进 行分析, 以理解高速列车底部流场特性, 其中根据流 场压力系数对数值模拟结果进行试验验证。

\section{1 试验验证}

考虑本列车模型尚无风洞试验数据, 采用其动 模型测试数据进行数值计算验证。试验在英国伯明 翰大学(University of Birmingham)列车动模型试验 线上进行 ${ }^{[17]}$, 通过发射装置将与数值计算相同的高 速列车模型弹射后, 使其沿着轨道线路运行, 列车 通过测点时运行速度为 $41.67 \mathrm{~m} / \mathrm{s}$ 。为了便于在道床 面与列车底部间安装测量流场压力与速度等物理量 的仪器与设备, 采用整体半松散介质模拟的碎石道 床被置于列车上方, 故列车车身需要翻转 $180^{\circ}$ 运行 通过道床区段(图 $4^{[17]}$ ), 方向朝向往下的车顶面通过滑

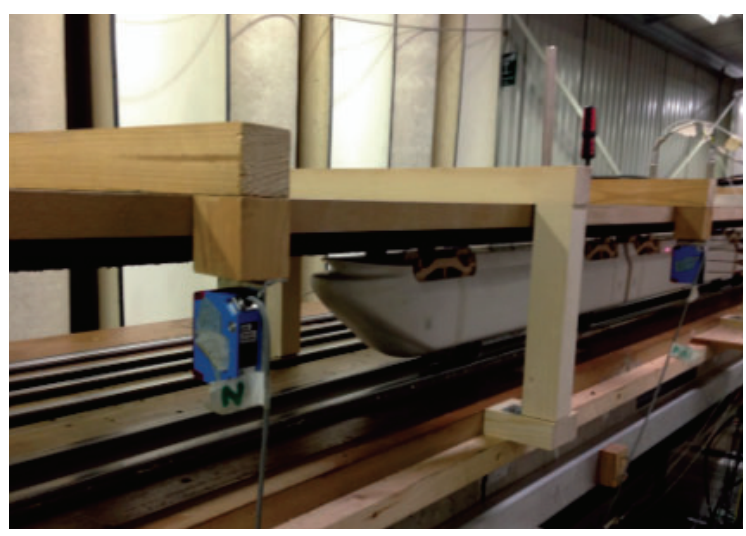

图 4 列车动模型试验
轮运行在试验线路上, 使得列车顶部与下方轨 面间保持一定间距, 以确保列车顶部流动发展具有 一定空间, 减少来自于地面影响。故动模型试验中, 列车车轮被固结在转向架上, 保持静止, 车轮底部 部分切除, 以平面形式滑行通过钢轨踏面, 与数值 模拟时增加轮轨接触面相一致。数值风洞模拟时列 车静止处于计算域内, 计算时沿列车纵向布置了一 系列等间距排列的测点, 位于距离钢轨顶面 $4 \mathrm{~mm}$ 的线路纵向中心线上，如图 5 所示。各测点所在间 距内得到的物理量信号按列车运行速度被依次连接 在一起，以获得列车通过线路时各物理量的时间历 程信号。各测点之间的间距越小，所获得物理量的 时间序列信号精度越高 ${ }^{[13]}$ 。根据该方法计算得到的 列车底部流场压力系数 $\left(C_{p}=\left(p-p_{0}\right)\right) /\left(\frac{1}{2} \rho_{0} U_{\infty}^{2}\right)$, 其中, $U_{\infty}$ 为来流速度, $p$ 为压力, $p_{0}$ 与 $\rho_{0}$ 为静止 流场的空气压力与密度)及其与试验数据的比较如 图 6 所示。考虑数值计算时列车静止, 在来流作用 下形成底部流动, 而动模型测试时由列车运行产生 底部流动。另外数值模拟时采用了平滑的刚性地面, 而试验中采用了模拟碎石道床的整体多孔介质材 料, 可以使得流体穿越通过, 影响了列车底部流场内 动量和压力分布。同时车身翻转 $180^{\circ}$, 列车顶部区 域地面结构将影响列车底部流场发展, 使得列车模型

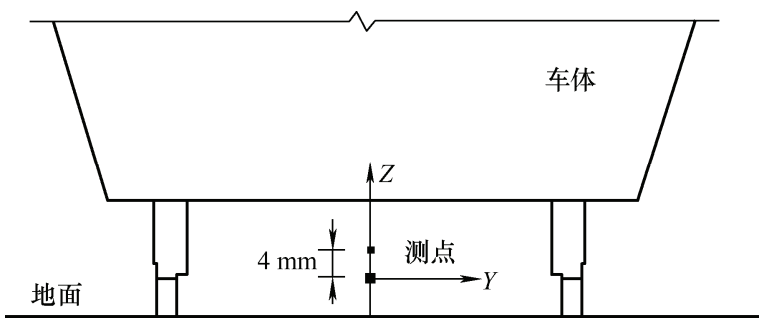

图 5 测点位置横截面图

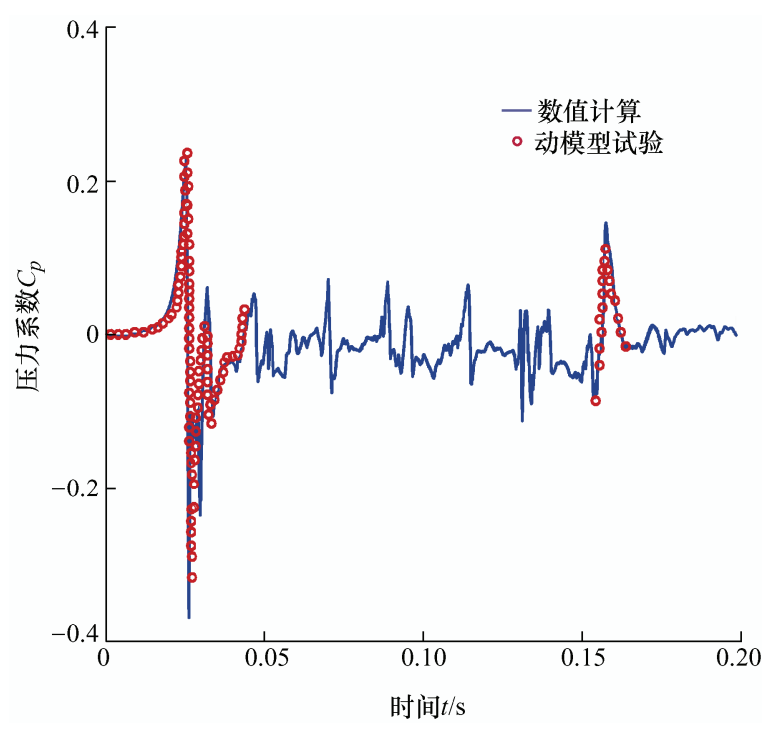

图 6 压力系数时间序列 
中间车区域底部流动影响因素复杂, 难于与仿真计 算值进行对比。故采用动模型试验中车头与车尾部 位通过时的试验数据进行数值计算验证。图 6 表明 数值模拟与测试结果吻合良好, 一定程度上验证了 数值计算准确性。另根据图 6 可见, 列车车头、各 车厢连接部位与车尾处产生了较强压力脉动, 尤其 是车头部位压力峰值最为显著, 该脉动压力作用在 道床面上, 伴随着列车风作用容易将道砟颗粒吹起。 因此, 车头部位鼻雉和排障器等底部结构进行流线 型设计, 可以降低车头转向架区域流动冲击作用, 减少道砟飞砟现象产生。

\section{2 列车底部流场特性分析}

根据上述流场内物理量时间历程计算方法, 三 方向速度系数(流向 $1-u / U_{\infty}$, 垂向: $u / U_{\infty}$, 展向: $w / U_{\infty}$, 其中 $u 、 v$ 与 $w$ 分别为流体流向、垂向与展 向速度分量)时间序列求解结果如图 7 所示, 其计算 位置与压力系数计算位置相同(图 5)。图 7a 绘出了 沿线路方向速度系数, 可以发现在车头鼻雉区域由 于驻点压力较高使得流向速度增加, 当流体沿与列

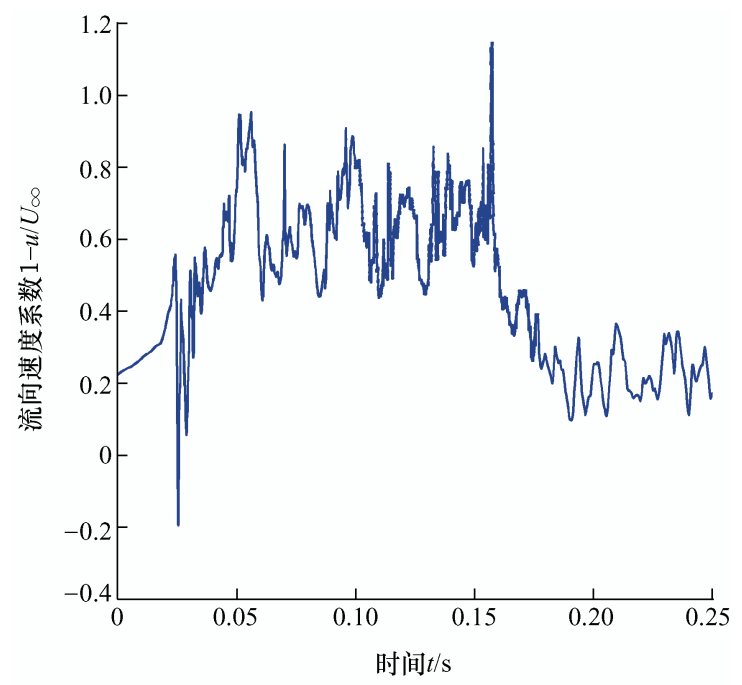

(a) 流向

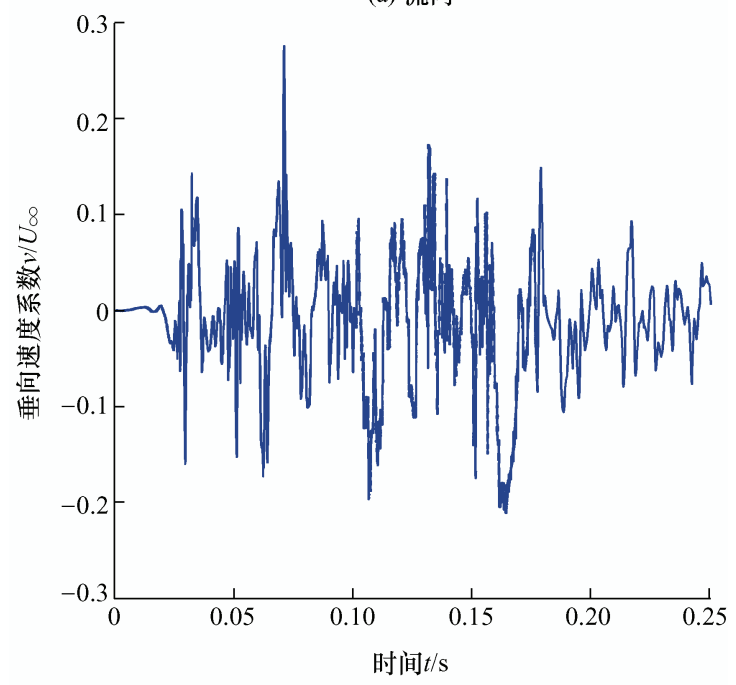

(b) 垂向

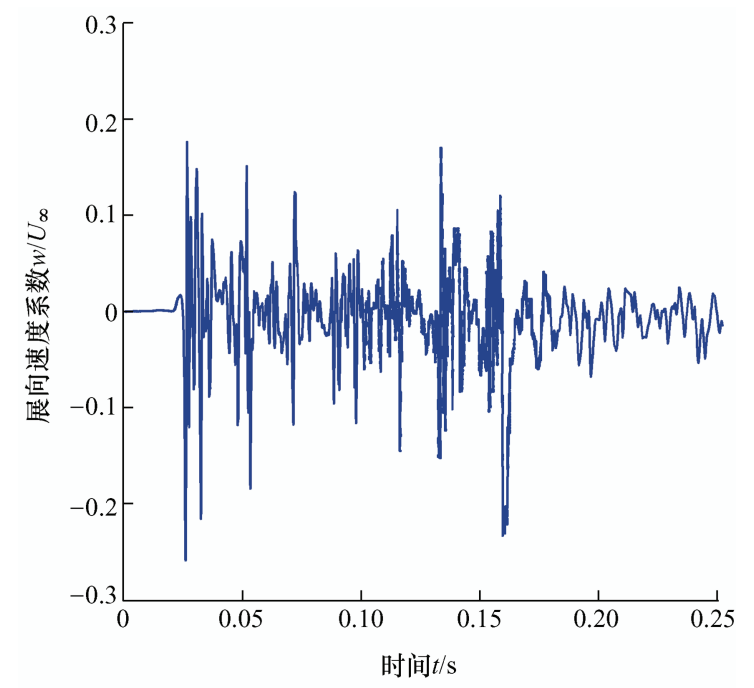

(c) 展向

图 7 速度系数时间序列

车运行反方向流动时, 流向速度则迅速下降, 然后流 体速度再次增加并在转向架舱和车厢连接部位脉动变 化。最大流向速度出现在车尾尾流区域, 这是由于列 车尾迹内大尺度流向浴的形成产生了较大的尾浴振 荡, 如下文所述。与流向速度相比, 流体垂向与展向 速度相对较小, 如图 $7 \mathrm{~b}$ 与图 7c 所示。因此, 有砟轨 道表面形成的以流向为主、具有较高扫掠速度的剪切 类流动极易将道砟颗粒带起, 诱发道砟飞砟现象产生。

高速列车周围流场中湍流浴结构如图 8 所示, 采 用 $Q$ 准则显示 $\alpha=30$ 的等值面, $\alpha=Q /\left[\left(U_{\infty} / H\right)^{2}\right]$, 其中 $Q$ 为速度梯度张量第二不变量; 灰度表示无量纲 化平均速度幅值的大小。图 $8 \mathrm{a}$ 显示了头车、第 2 车和 第 3 车(前半车)转向架和车厢连接处等部位的瞬态浴结 构, 可以发现车头排章器和转向架部位, 由于前方来 流的流动冲击作用, 生成了较高湍流度流场, 涡流特 性显著。流体在车头底部排障器与转向架舱前缘分离 后与转向架各部件相互作用, 随后撞击转向架舱后缘 壁面, 致使转向架周围区域流动变化剧烈。由图 8a $8 \mathrm{c}$ 可见, 各车厢连接处紧邻着前后转向架或位于铰接 式转向架上方, 使得该区域存在复杂的表面结构不连 续, 流动发展受到几何体干扰, 导致流动冲击作用较 强; 流体沿车长方向流经各个转向架时, 在转向架尾 涡沿展向振荡作用下，车体底部形成大量不同尺度的 湍浴向下游传播, 并沿车体侧向浴流分布逐渐增宽, 流动中包含大量不同方向的小尺度浴结构, 使得列车 后半部分转向架区域产生的湍涡较前半部分(除头车以 外)逐渐增多; 与车头转向架区域流动相比, 中间车各 转向架区域的流体相互作用较弱; 另外与普通转向架 区域相比, 铰接式转向架处流动变化较强、湍浴发展 较多。图 $8 \mathrm{c}$ 显示列车尾端转向架周围产生的湍涡与列 车尾流相互作用、相互融合, 形成了复杂的尾涡流场。 
因此, 列车车头底部排障器、各转向架与车厢连接处 以及车尾底部等区域的流动变化显著, 这些部位高速
通过道床面时, 将对道床面产生剪切类流动作用, 并 对道砟颗粒产生较强的气动力作用。

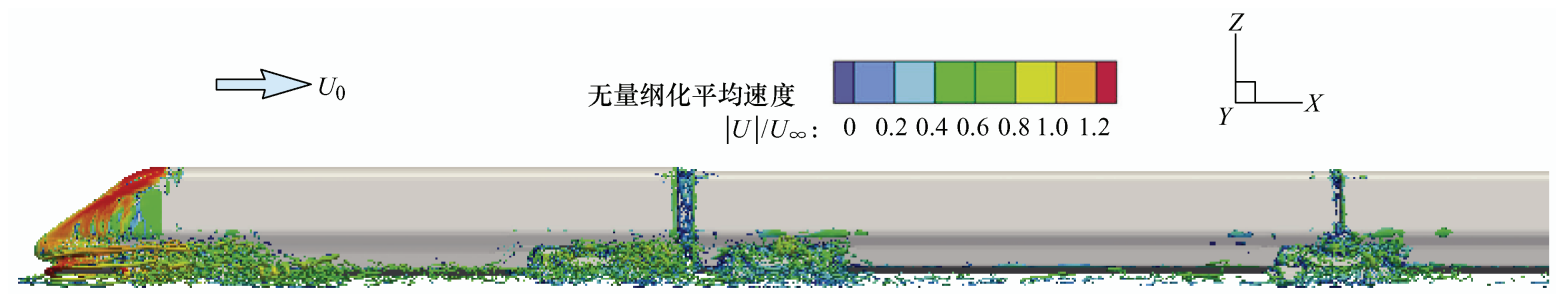

(a) 列车头车区域各转向架部位 (头车与第2、3节车)

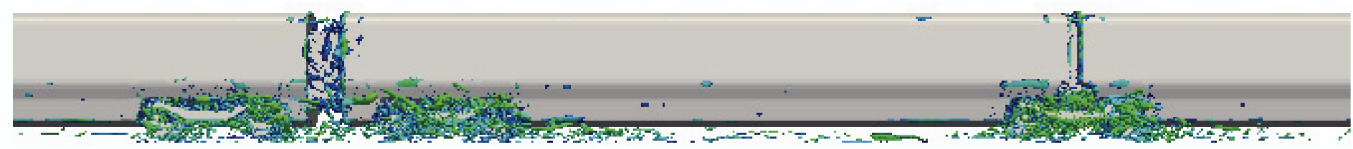

(b) 列车中间车各转向架部位(第3、4、5节车)

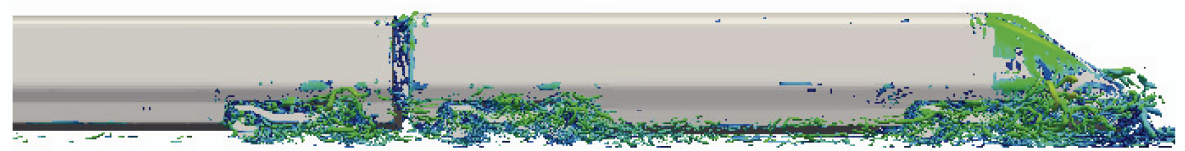

(c) 列车尾端各转向架部位(第5节车与尾车)

图 $8 Q$ 准则显示列车周围涡结构

由于流向浴可以较好表明列车周围流动发展, 根据图 9 显示的沿列车高度方向的 3 个水平截面位置 $(z=0.006 \mathrm{~m} 、 0.026 \mathrm{~m} 、 0.108 \mathrm{~m})$, 图 10 绘出了相应 3 截面上列车周围瞬态涡量场轮廓图 $\left(\omega_{x}=(\partial w / \partial y-\partial v / \partial z) H / U_{\infty}\right)$ 。由图 10a 可见, 列车 底部各转向架舱浴脱落在转向架尾迹区产生两条狭 长的浴量, 形成沿流向的两平行特征线, 其间距与 轮间距相近; 同时车体底部结构流动与地面边界 层相互作用, 产生了不同尺度旋浴, 在列车底部 区域阻塞效应影响下沿列车车长方向逐渐向展 向扩展, 最后在尾流区交汇, 导致列车尾迹展向
宽度逐渐增加。分析图 $10 \mathrm{~b}$ 可以发现, 各转向架舱 近地空腔结构内由于流动分离与涡流作用, 产生了 密集分布的湍浴, 浴量强度较大; 车体侧面转向架 舱区域浴脱落后浴流向下游传播, 与列车尾流相互 作用生成了一对流向浴, 沿展向振荡剧烈。图 10c 显示列车周围流动变化在车体流线型外表面变得 逐渐平缓, 浴量强度沿列车高度方向逐渐减小。因 此, 高速列车底部区域内流动变化剧烈, 特别是转 向架舱和车厢连接处流体发展极不稳定, 强烈的气 流冲击作用在有砟轨道道床面上, 容易导致飞砟产 生。

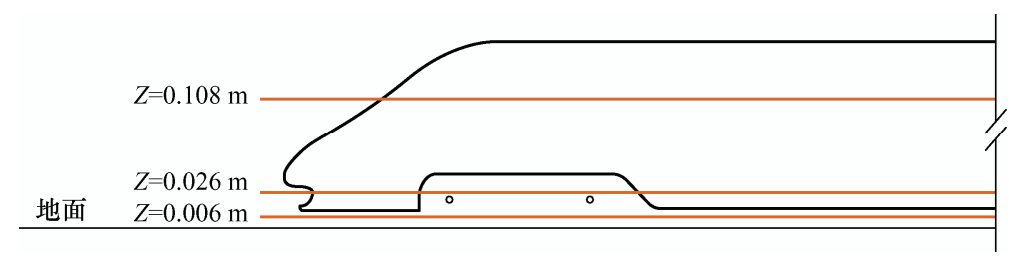

图 9 沿车体高度方向水平切面位置
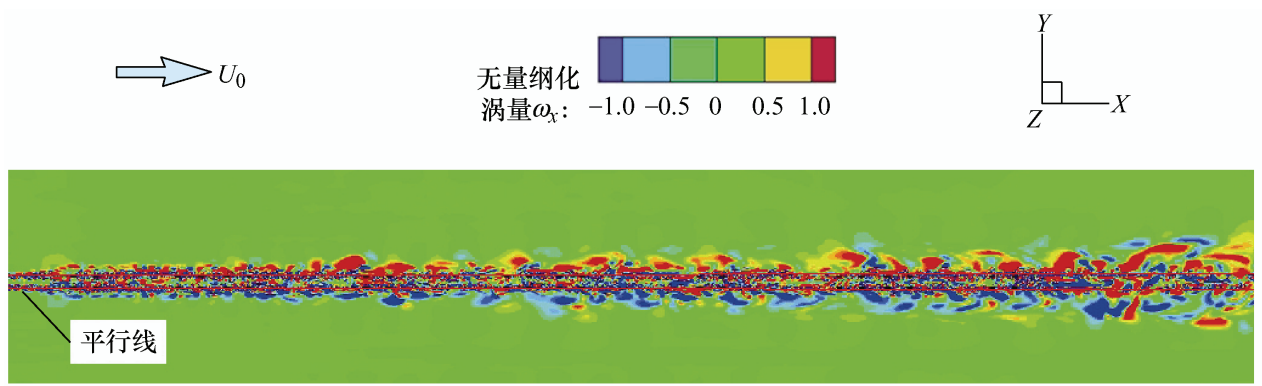

(a) $z=0.006 \mathrm{~m}$ 


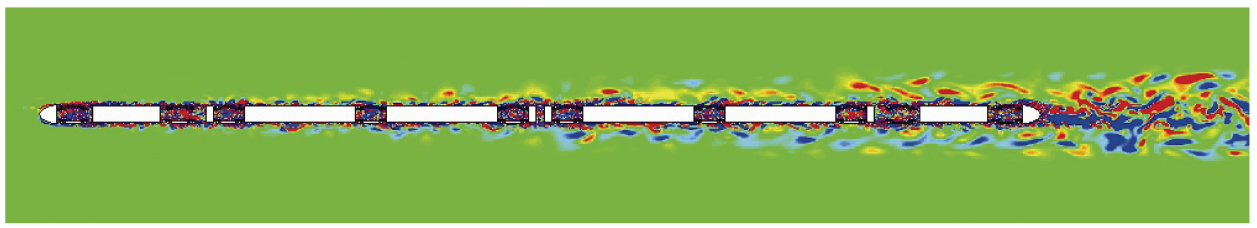

(b) $z=0.026 \mathrm{~m}$

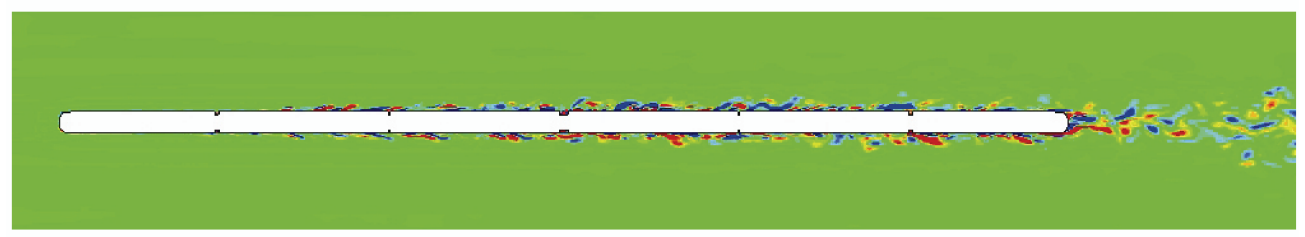

(c) $z=0.108 \mathrm{~m}$

图 10 列车周围不同高度瞬态涡量场

另外, 采用湍流强度 $\left(u^{\prime} / U_{\text {mean }}\right.$, 其中 $u^{\prime}$ 是流场速 度均方根值, $U_{\text {mean }}$ 为流场平均速度)分析列车底部 流动湍流发展水平。列车底部流场观察点位置如图 11 所示, 沿车体侧向从列车中心线至车体外侧沿车长方 向共有 6 个断面, 图 12 显示了不同断面处 3 个高度 上列车底部流动湍流强度, 可以发现在这些观测位 置中, 除了远离转向架区域的车体外侧断面 $(y=0.07 \mathrm{~m})$ 外, 最大湍流强度值均位于车头附近区域, 显然与车头部位较强的流动冲击有关, 然后沿车长方 向随车间距和列车尾端位置呈现规律性脉动变化, 最 后远离车尾一定距离后逐渐衰减, 如图 12a 12e 所示; 对于距离车体较远部位 $(y=0.07 \mathrm{~m})$, 最大湍流度出现在 尾流区域, 如图 12f 所示, 但强度较弱, 主要是由于受 到了列车尾迹内展向周期性振荡浴运动对于近地区域 产生的流动干扰。因此, 列车底部流动的湍流强度分 布再次表明车头及其转向架区域流动变化剧烈, 对道 床面道砟颗粒的气动干扰作用较大。分析列车底部流 动在垂向方向的湍流强度变化发现, 在列车中心线 附近区域, 流动相互作用强烈、湍流发展充分, 使

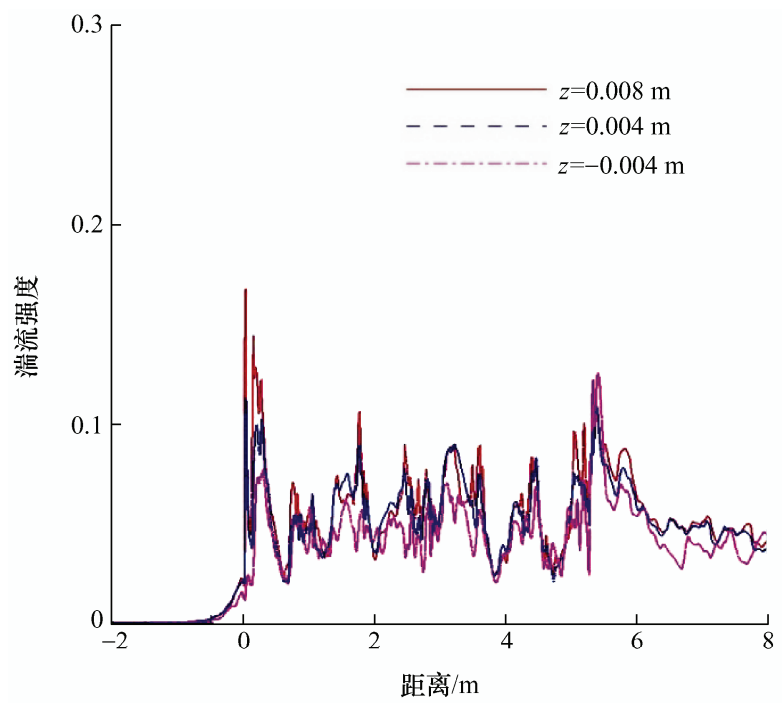

(a) $y=0 \mathrm{~m}$
得沿高度方向湍流度分布相似, 如图 12a、12b 所示。 根据图 $12 \mathrm{c} \sim 12 \mathrm{e}$ 可见, 在远离列车中心线的轮对周 围区域, 靠近地面 $(z=-0.004 \mathrm{~m})$ 的列车底部流场湍 流度较低, 但随着高度增加, 湍流度增长较快, 在 轨面以上区域湍流度较高、幅值与分布特性较为相 似。由此可见, 轮对周围靠近地面流动受到车体底 部结构与地面影响, 发展受到限制, 湍流强度较低, 但沿垂向伴随着车体底部流动发展加快, 湍流度迅 速增加, 使得在轮轨耦合振动作用下离开道床面的 道砟颗粒容易进入湍流度较高的车体底部流动区 域, 在流场脉动力作用下产生飞砟并散落在轨面上, 导致车辆驶过后产生轮轨面伤损。

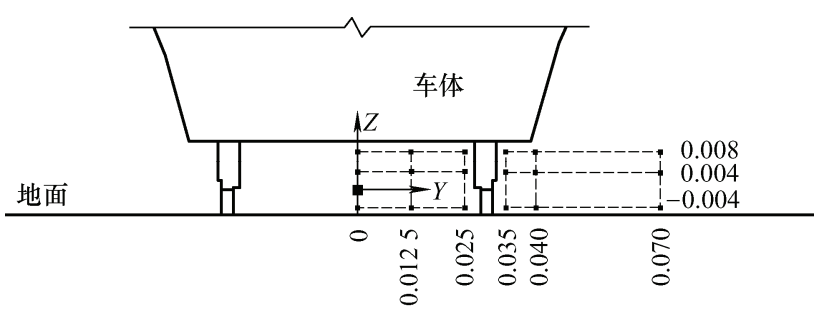

图 11 列车底部流场观察点位置 $(\mathrm{m})$

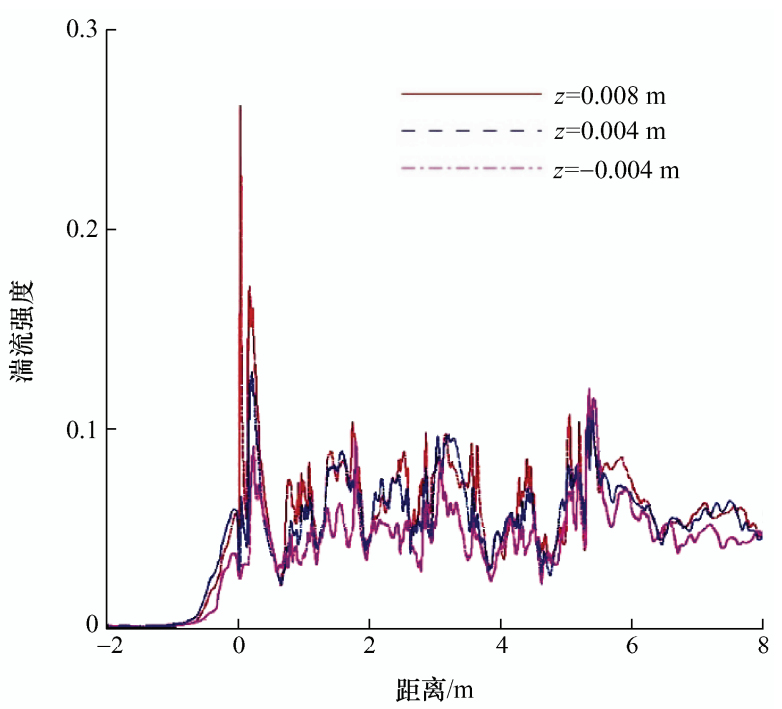

(b) $y=0.0125 \mathrm{~m}$ 


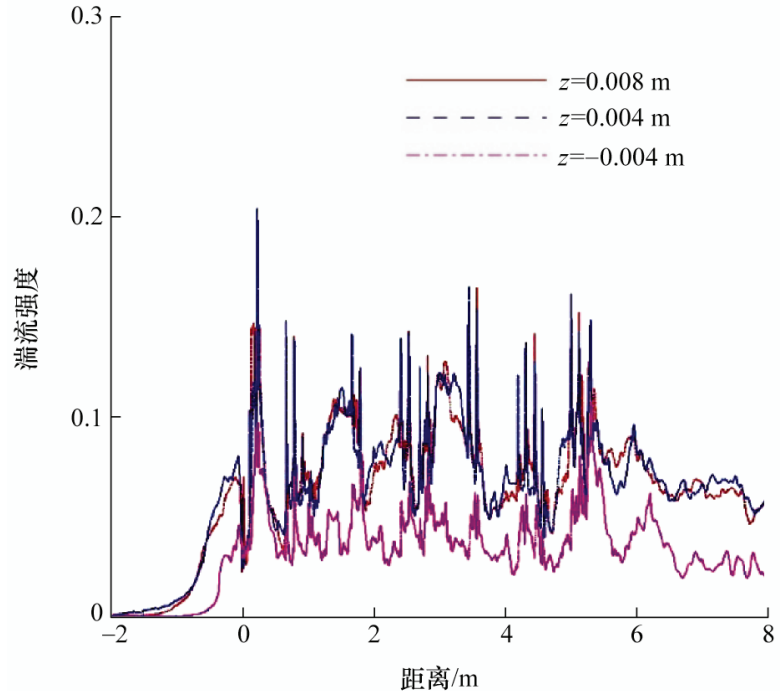

(c) $y=0.025 \mathrm{~m}$

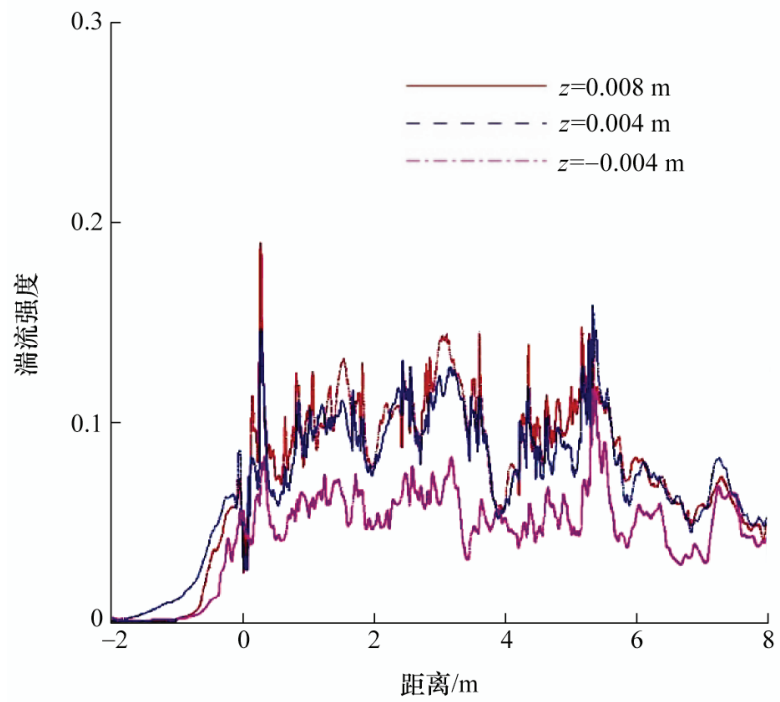

(e) $y=0.04 \mathrm{~m}$

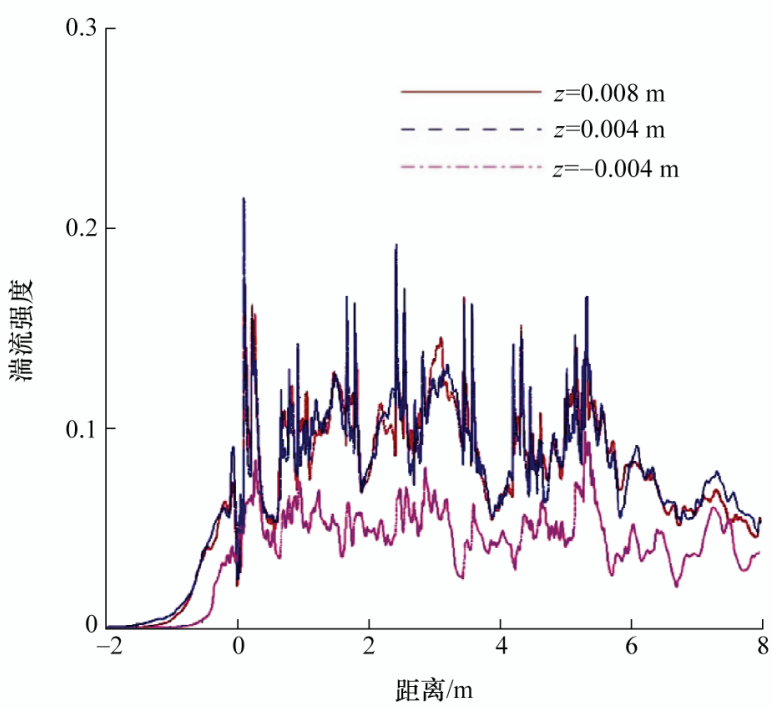

(d) $y=0.035 \mathrm{~m}$

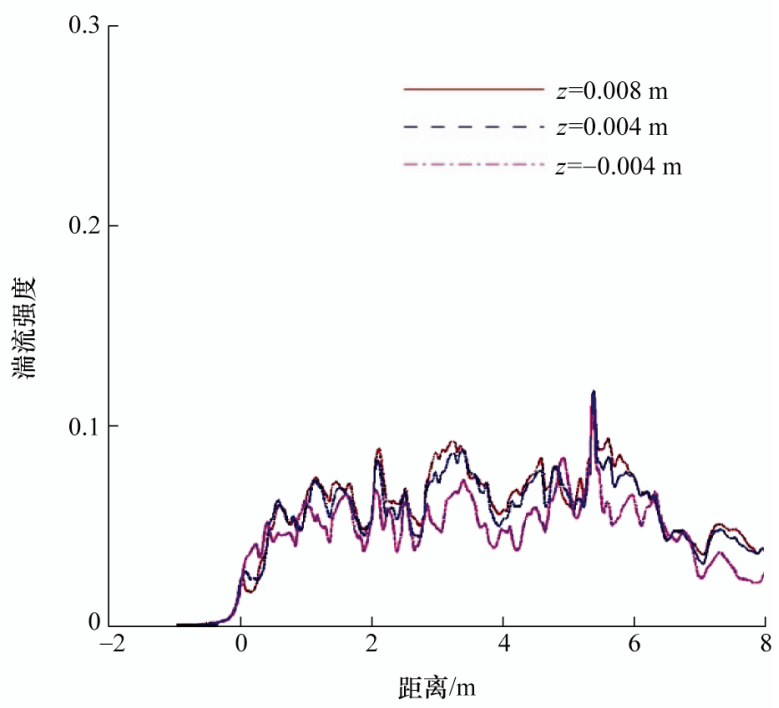

(f) $y=0.07 \mathrm{~m}$

图 12 沿列车方向车底流动的湍流强度

在距离列车尾部 $1 H$ ( $H$ 为列车高度)与流向垂 直的切面上, 列车尾迹内形成的呈对称分布反向旋 转的流向浴的平均流线如图 13 所示, MULD 等 ${ }^{[20]}$

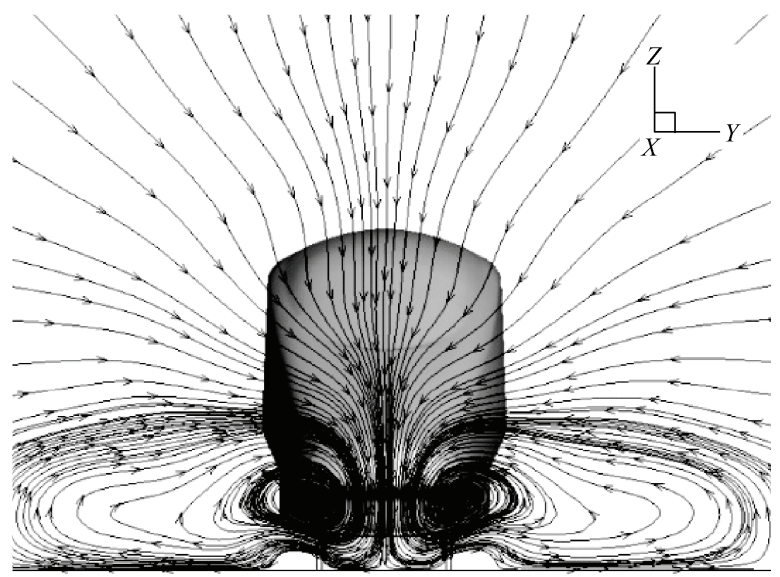

图 13 列车尾部尾流附近的平均流线 ( $Y-Z$ 平面)
通过研究也描述了在列车尾流内产生的这对流 向浴。由图 13 可见, 列车尾迹内主流向浴对沿 尾流向车后发展, 从轨道中心线逐渐向列车两侧 与底部线路运动, 伴随着大尺度尾浴形成的强剪 应力与道床面产生相互作用。因此, 当具有较大 流动能量的尾流扫掠过道床表面时, 会将道砟颗 粒抛射到钢轨踏面上, 从而当后续列车通过时, 造成轮轨间碾压伤损。

\section{3 转向架气动性能分析}

根据以上高速列车转向架区域周围流场分 析可知, 转向架类型将直接影响车体底部流动性 能, 另外与普通转向架相比, 铰接式转向架周围 流场将更为复杂, 故对转向架气动力特性进行分 析。图 14 比较了各转向架(第 $1 、 2 、 4$ 位与车尾 处转向架)气动力系数功率谱密度(PSD), 其中第 
4 位转向架为铰接式转向架, 气动力系数定义为转 向架所受升力、阻力和侧向力, 通过 $\frac{1}{2} \rho_{0} U_{\infty}^{2} A$ (其 中 $A$ 为车体正投影面积)进行无量纲化。分析图 14 可见, 在 $100 \mathrm{~Hz}$ 以上频率范围内, 车头转向架(第 1 位转向架)各气动力系数最高, 这是由于车头转 向架周围强烈的流动冲击作用在转向架上产生 了较大的脉动力, 另外, 铰接式转向架(第 4 位转 向架)和车尾处转向架所受气动力的频谱特性和 幅值相似, 在低于 $100 \mathrm{~Hz}$ 频域内, 该两转向架 的侧向力系数远高于其余两转向架, 说明由于铰 接式转向架部位车厢连接处与转向架舱涡流相 互作用以及车尾处旋浴脱落与尾浴振荡激扰, 两 部位转向架分别受到了较强的侧向流动力作用, 将会直接影响这些区域道床面道砟颗粒的受力 状态。

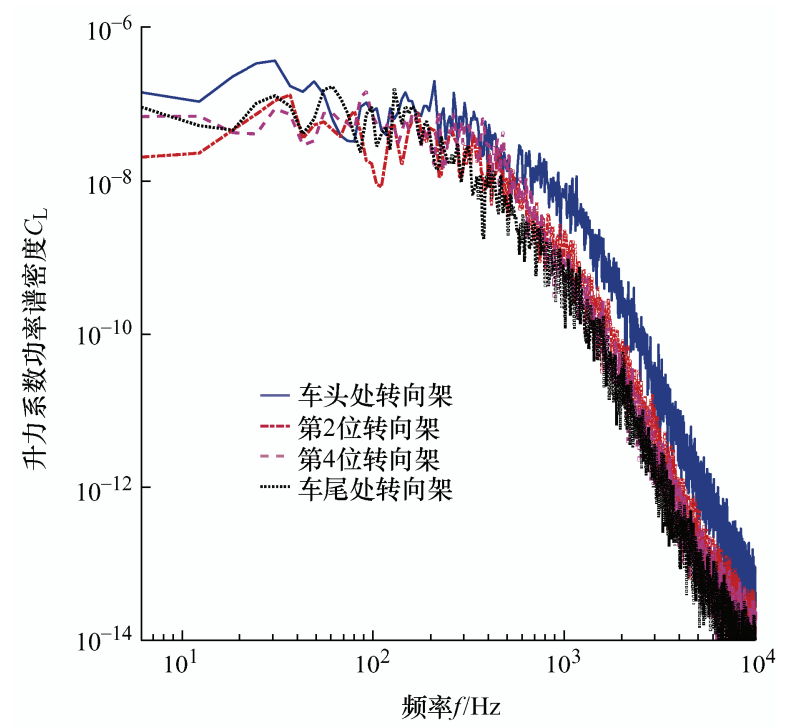

(a) 升力系数

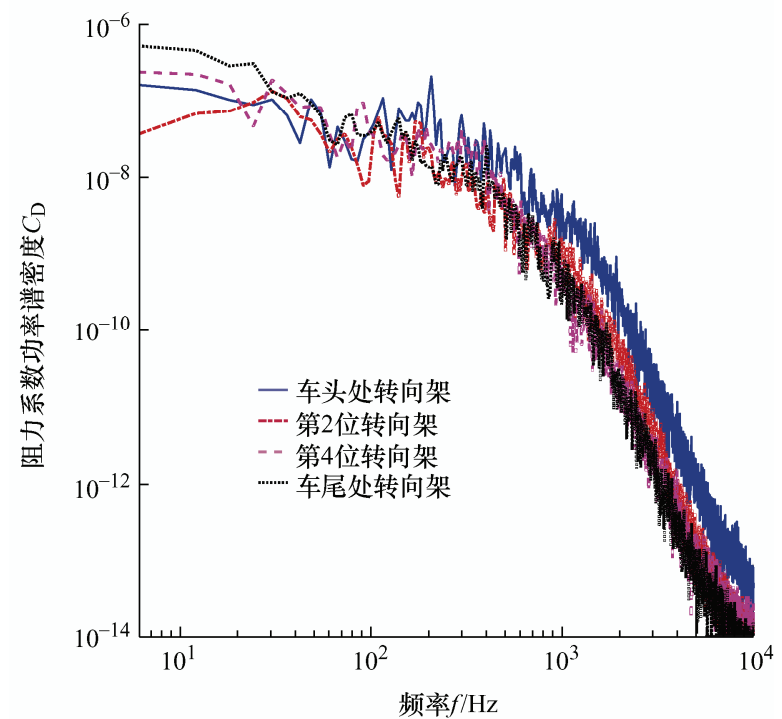

(b) 阻力系数

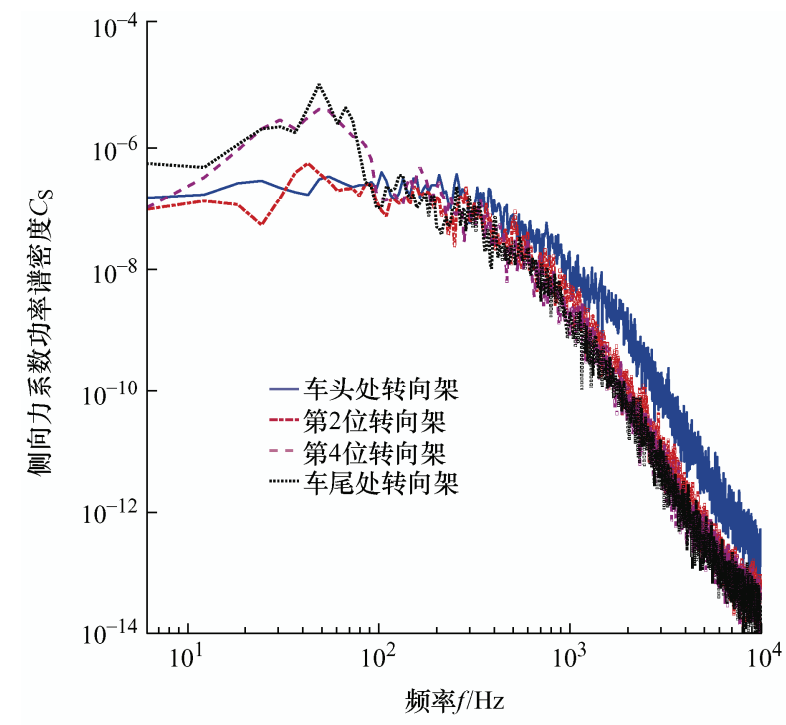

(c) 侧向力系数

图 14 不同转向架的力系数的功率谱密度

为进一步分析各转向架气动特性, 图 15 显示了位 于车头、第 2 位、铰接式(第 4 位)和车尾转向架共 4 部 位处流场观察点的压力系数与速度系数 $\left(u / U_{\infty}, u\right.$ 为 沿 $x, y$ 和 $z$ 方向的速度分量: $u_{x} 、 u_{y}$ 与 $u_{z}$ ) 的功率谱密 度。如前所述, 由于头车前转向架区域受到来流较强 冲击, 车头转向架上压力与速度脉动均高于其余转向 架, 特别是压力脉动增幅显著, 这与图 6 所示的车头 部位形成的强压力脉动相关联。比较车头转向架以外 的 3 个转向架可知, 车尾转向架的速度脉动较大而较 接式转向架上的压力脉动较大。究其原因, 车尾处转 向架区域浴脱落与尾流形成的尾浴相互作用, 尾流内 流向涡的振荡与扩散使得该转向架所受流场扰动作用 增加, 从而表现出频域内速度脉动幅值变大; 而铰接 式转向架处两车间隙内的流动分离与涡流作用直接影 响了该处转向架周围动量传递, 导致其所受压力增加。

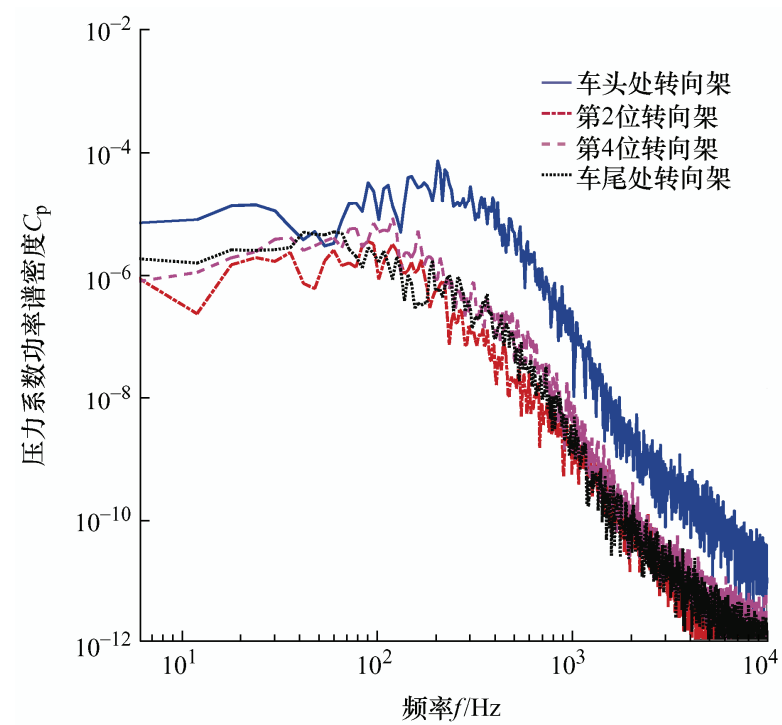

(a) 压力系数 


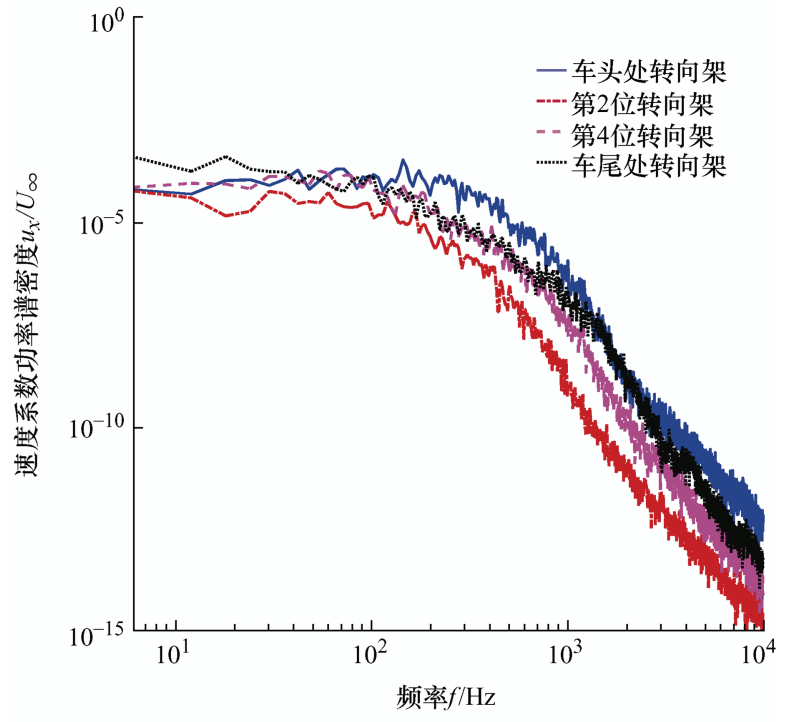

(b) 速度系数 $\left(u_{x}\right)$

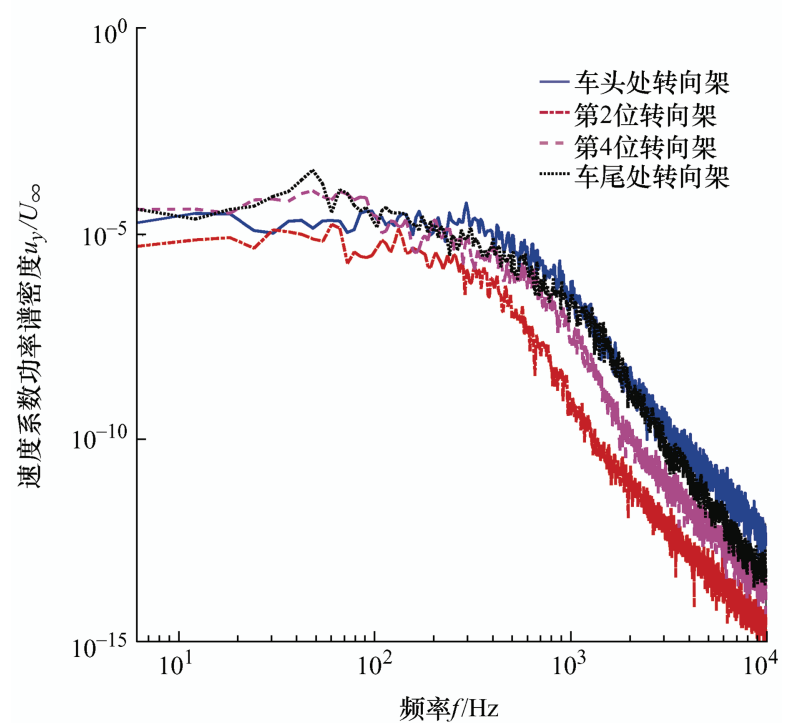

(c) 速度系数 $\left(u_{y}\right)$

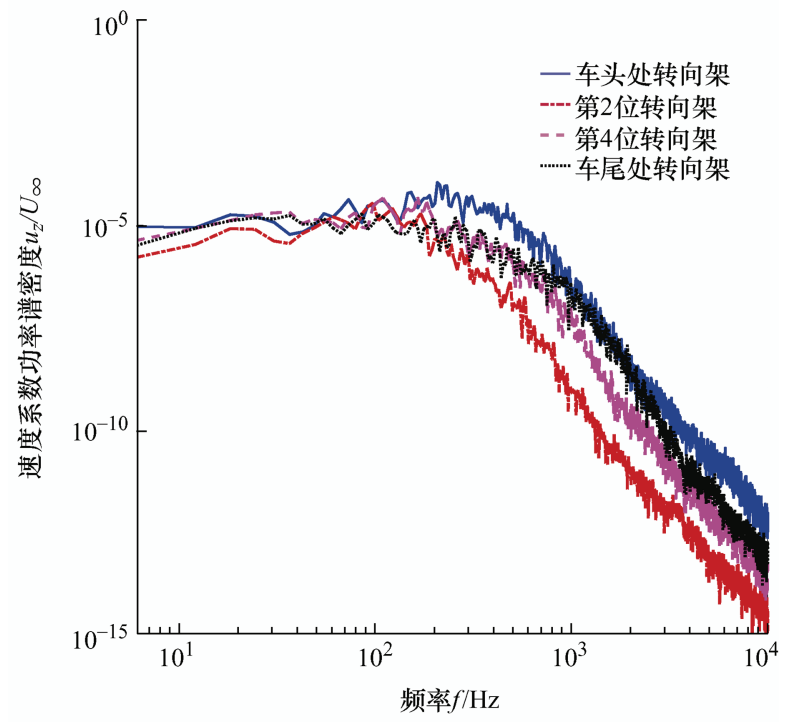

(d) 速度系数 $\left(u_{z}\right)$

图 15 列车底部流场观察点压力和速度系数的功率谱密度

\section{4 结论}

本文基于延迟分离浴模型，计算分析了高速列 车底部流动特性。结果表明, 列车周围产生的湍浴 相互作用并沿车体往下游传播, 形成复杂的非定常 流动, 湍流强度较高。位于钢轨踏面上方的列车底 部流动受地面影响较小, 湍流发展充分, 其强度高 于位于钢轨踏面以下流动, 使得道砟颗粒在轮轨动 力作用下振离道床面后, 在车体底部流场脉动力作 用下形成飞砟。列车车头、排障器, 转向架与车厢 连接处等部位产生了较强流动分离, 形成较大压力 峰值, 尤其车头部位产生剧烈压力脉动; 由于铰接 式转向架座落于车厢连接处的车体表面不连续部 位, 在转向架舱内产生了较强流体相互作用。这些 区域高速通过道床面时, 容易产生道砟飞砟现象, 故车头流线型和车厢连接处表面平顺等措施可以 缓和高速列车关键区域高湍流度流动对于道床面 的扰动作用。列车尾迹内尾浴流动能量较大, 该浴 流下洗扫掠道床面时, 易将道砟颗粒抛射至钢轨踏 面上, 使其受到后继运行列车车轮的碾压, 造成车 轮扁疮和钢轨压溃等伤损，因此控制列车尾流发 展, 减弱列车风生成与影响, 将有利于减少道砟飞 砟现象发生。

\section{参 考 文 献}

[1] 田红旗. 中国高速轨道交通空气动力学研究进展及发 展思考 [J]. 中国工程科学, 2015 (4): 30-41.

TIAN Hongqi. Development of research on aerodynamics of high-speed rails in China[J]. Engineering Sciences,

2015(4): 30-41.

[2] 杨国伟, 魏宇杰, 赵桂林, 等. 高速列车的关键力学问 题[J]. 力学进展, 2015, 45: 217-459.

YANG Guowei, WEI Yujie, ZHAO Guilin, et al. Research progress on the mechanics of high speed rails $[\mathrm{J}]$. Advances in Mechanics， 2015， 45: 217-459.

[3] 梁习锋, 田红旗. 列车气动性能评估参数研究 [J]. 中国 铁道科学, 2003, 24(1): 38-42.

LIANG Xifeng, TIAN Hongqi. Research on evaluating parameters of train aerodynamics performance[J]. China Railway Science, 2003, 24(1): 38-42.

[4] BAKER C J. The flow around high speed trains[J]. Journal of Wind Engineering and Industrial Aerodynamics, 2010, 98 : 277-298.

[5] SCHETZ J A. Aerodynamics of high speed trains[J]. 
Annual Review of Fluid Mechanics, 2001, 33: 371-414.

[6] QUINN A D, HAYWARD M, BAKER C J, et al. A full-scale experimental and modelling study of ballast flight under high-speed trains[J]. Proceedings of the Institution of Mechanical Engineers Part F-Journal of Rail and Rapid Transit, 2009, 224: 61-74.

[7] GARCIA J, CRESPO A, BERASARTE A, et al. Study of the flow between the train underbody and the ballast $\operatorname{track}[\mathrm{J}]$. Journal of Wind Engineering and Industrial Aerodynamics, 2011, 99: 1089-1098.

[8] 孙振旭, 郭迪龙, 姚远, 等. 高速列车地面效应数值模 拟研究 $[\mathrm{J}]$. 计算物理, 2013, 30(1): 61-69.

SUN Zhenxu, GUO Dilong, YAO Yuan, et al. Numerical study on ground effect of high speed trains[J]. Chinese Journal of Computational Physics, 2013, 30(1): 61-69.

[9] ZHANG J, LI J J, TIAN H Q, et al. Impact of ground and wheel boundary conditions on numerical simulation of the high-speed train aerodynamic performance[J]. Journal of Fluids and Structures, 2014, 228(1): 25-36.

[10] BELL J R, BURTON D, THOMPSON M, et al. Wind tunnel analysis of the slipstream and wake of a high-speed train[J]. Journal of Wind Engineering and Industrial Aerodynamics, 2014, 134: 122-138.

[11] PREMOLI A, ROCCHI D, SCHITO P, et al. Ballast flight under high-speed trains : Wind tunnel full-scale experimental tests[J]. Journal of Wind Engineering and Industrial Aerodynamics, 2015, 145: 351-361.

[12] ZHU J Y, HU Z W. Flow on the ballasted trackbed with permeable surfaces and its influence on the ballast flight $[\mathrm{C} / \mathrm{CD}] / /$ The 4 th International Conference on Road and Rail Infrastructure, Croatia, 2016.

[13] ZHU J Y, HU Z W. Flow between the train underbody and trackbed around the bogie area and its impact on ballast flight[J]. Journal of Wind Engineering and
Industrial Aerodynamics, 2017, 166: 20-28.

[14] WANG S B, BURTON D, HERBST A H, et al. The effect of the ground condition on high-speed train slipstream[J]. Journal of Wind Engineering and Industrial Aerodynamics, 2018, 172: 230-243.

[15] SPALART P R, DECK S, SHUR M L, et al. A new version of detached-eddy simulation, resistant to ambiguous grid densities[J]. Theoretical and Computational Fluid Dynamics, 2006, 20: 181-195.

[16] SPALART P R, ALLMARAS S R. A one-equation turbulence model for aerodynamic flows[J]. AIAA Paper 92-0439, 1992.

[17] SOPER D, FLYNN D, BAKER C, et al. A comparative study of methods to simulate aerodynamic flow beneath a high-speed train $[\mathrm{J}]$. Proceedings of the Institution of Mechanical Engineers Part F: Journal of Rail and Rapid Transit, 232, 2018, 1464-1482.

[18] European Committee for Standardization(CEN). Railway applications-Aerodynamics Part 4: Requirements and Test Procedures for Aerodynamics on Open Track EN14067-4[S], British: CEN, 2013.

[19] OZA U, HU Z W, ZHANG X. Effect of cavity flow on landing gear aerodynamic loads[R]. AIAA, 2015-2288, 2015.

[20] MULD T W, EFRAIMSSON G, HENNINGSON D S. Wake characteristics of high-speed trains with different lengths[J]. Proceedings of the Institution of Mechanical Engineers Part F: Journal of Rail and Rapid Transit, 2014, 228(4): 333-342.

作者简介: 朱剑月, 男, 1973 年出生, 博士, 副教授, 博士研究生导师。 主要研究方向为高速列车空气动力学与气动噪声等。

E-mail: zhujianyue@tongji.edu.cn

陈力(通信作者), 女, 1979 年出生, 博士研究生, 高级工程师。主要研 究方向为风洞测试技术与科技平台管理等。

E-mail: lilychen@tongji.edu.cn 